\title{
Dynamics of the global water cycle of Biosphere 2
}

\author{
Francesco N. Tubiello a,*, John W. Druitt ${ }^{\mathrm{b}}$, \\ Bruno D.V. Marino ${ }^{\mathrm{c}}$ \\ a Columbia University and NASA-Goddard Institute for Space Studies, 2880 Broadway, New York, \\ NY 10025, USA \\ ${ }^{\mathrm{b}}$ Biosphere 2 Center for Research and Education, Highway 77, PO Box 689, Oracle, AZ 85623, USA \\ ${ }^{\mathrm{c}}$ Department of Earth and Planetary Sciences, Harvard University, 20 Oxford St., Cambridge, \\ MA 02138, USA
}

Received 2 October 1996; received in revised form 15 October 1997; accepted 22 December 1997

\begin{abstract}
We investigate water usage and movement throughout the major reservoirs of Biosphere 2, Oracle AZ, over a period of 2 months. The data analyzed show reservoir turnover times ranging from hours to several years and the existence of three major water sub-cycles. A 'fast pool', or $60 \%$ of the available water used daily in Biosphere 2 , is recycled within a month through the air-handler's condensation system, as a consequence of plant and soil evapotranspiration; a 'medium pool', or $30 \%$ of available water, moves through the soil profile as drainage and is recycled within a year; while a 'slow pool,' or the remaining $10 \%$, moves through the Biosphere 2 ocean and takes several years to turnover. We develop a simple model of the Biosphere 2 water cycle and discuss its utility in estimating global and single-biome ecosystem processes within this facility. (C) 1999 Elsevier Science B.V. All rights reserved.
\end{abstract}

Keywords: Biosphere 2; Water cycle; Modeling

\section{Introduction}

The water system of Biosphere 2, Oracle AZ, was designed to support human and ecosystem survival under conditions of long-term mass closure (Petersen et al., 1992; Zabel et al., 1999). It integrates mechanical and natural systems to provide internal circulation for a vast amount of water, in the order of 6-7 million 1. More

\footnotetext{
* Corresponding author.
}

0925-8574/99/\$ - see front matter (C) 1999 Elsevier Science B.V. All rights reserved.

PII S0925-8574(98)00105-0 
than $80 \%$ of this water is exchanged rather slowly with the rest of Biosphere 2 water pool, residing in two large reservoirs: the Biosphere 2 ocean-marsh system; and the soil system. The remaining $20 \%$ is quickly circulated between the soil-plant-atmosphere system of Biosphere 2 and two major storage reservoirs: the primary storage, located in the south lung; and a network of storage tanks located in the basement of the agricultural (Marino et al., 1999) and the wilderness biomes of Biosphere 2: rain forest (Leigh et al., 1999), desert and savannah. Less than 1.5\% of total water resides at any time in the Biosphere 2 atmosphere, its streams and in additional tanks originally built for waste-water processing but currently not in use.

Detailed technical descriptions of the Biosphere 2 water cycle have been presented before (e.g. Dempster, 1992). We focus our attention to the following questions: How much of total Biosphere 2 water actually moves around the system on a daily basis? What are its preferred pathways and associated turnover times? With the aid of data collected during the period of November-December 1995 and by using a simplified model of Biosphere 2 water cycle, we present a first quantitative analysis of mean daily water usage and fluxes inside the system.

\section{The global water budget}

A likely estimate of Biosphere 2 total water pool is $6 \times 10^{6} 1$, with the largest uncertainty $\left( \pm 1 \times 10^{6} 1\right)$ regarding the water content in the soil system (Scalborough, Pers. Comm., 1995). The Biosphere 2 ocean-marsh system is by far the largest reservoir $\left(4 \times 10^{6} 1\right)$, followed by the soil $\left(1-2 \times 10^{6} 1\right)$; primary storage $\left(8 \times 10^{5} 1\right)$; and condensate tanks $\left(1.6 \times 10^{5} 1\right)$. All other reservoirs, including the Biosphere 2 pond and stream system $\left(5 \times 10^{4} 1\right)$ and the atmosphere $\left(<3 \times 10^{3} 1\right)$, have sizes that are at least one order of magnitude smaller than those discussed here and do not contribute significantly to the mass balance calculations (Table 1). As illustrated in Fig. 1, a simple model of the Biosphere 2 global water cycle can be constructed by considering only five reservoirs: ocean-marsh $(\mathrm{O})$; soil $(\mathrm{S})$; primary storage (PS); condensate tanks (TK); and the atmosphere (A). Biosphere 2 total water pool $(\Sigma \mathrm{W})$ can then be approximated by: $\Sigma \mathrm{W} \sim \mathrm{O}+\mathrm{S}+\mathrm{PS}+\mathrm{TK}+\mathrm{A}$. Daily fluxes among these reservoirs satisfy a simple mass-conservation law:

Table 1

Estimate of Biosphere 2 water reservoirs, in liters

\begin{tabular}{lcc}
\hline Reservoir & Mean estimate & $\%$ Of total \\
\hline Ocean-marsh & $4 \times 10^{6}$ & 61.3 \\
Soil & $1-2 \times 10^{6}$ & 23.0 \\
Primary storage & $8 \times 10^{5}$ & 12.3 \\
Condensate Tanks & $1.6 \times 10^{5}$ & 2.5 \\
Streams and other reservoirs & $5 \times 10^{4}$ & 0.8 \\
Atmosphere & $2 \times 10^{3}$ & 0.03 \\
Total & $6-7 \times 10^{6}$ & 100 \\
\hline
\end{tabular}




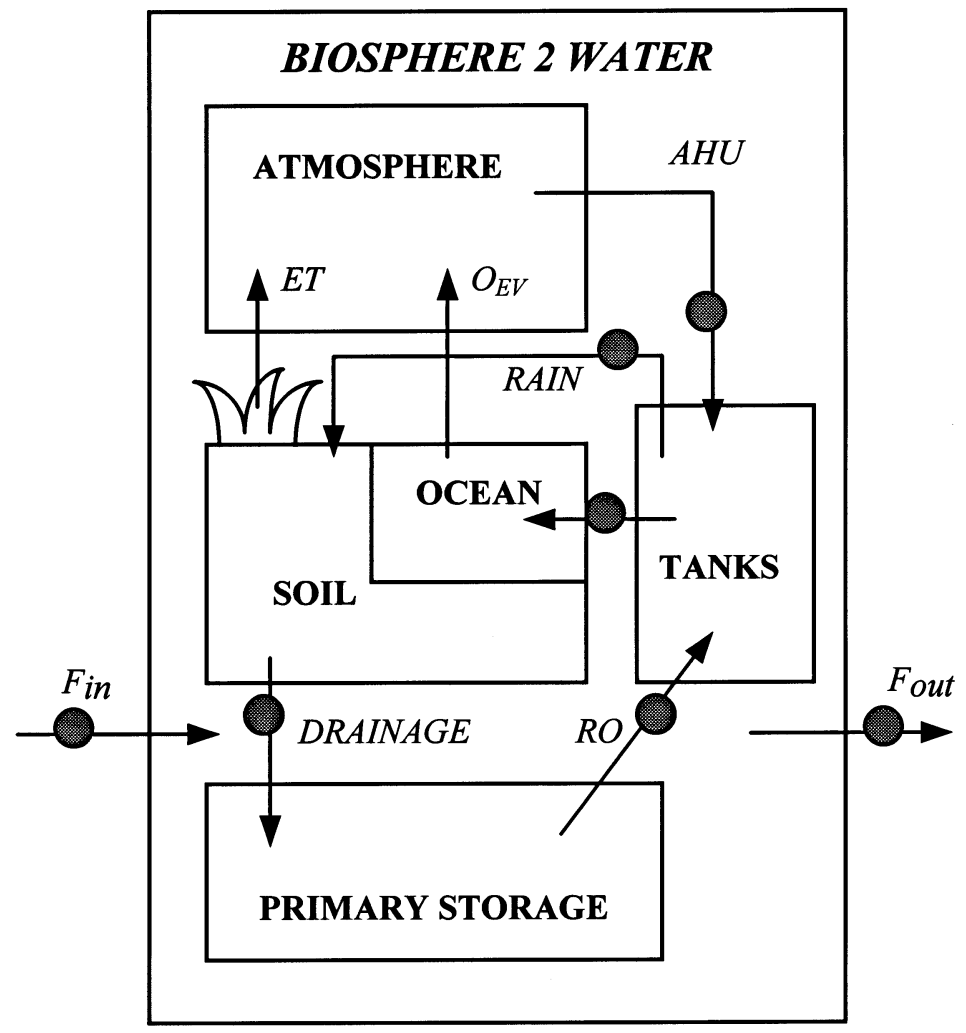

Fig. 1. A simplified view of Biosphere 2 global water cycle. Circles indicate measured water fluxes.

$$
\Delta \Sigma \mathrm{W} \sim \Delta \mathrm{O}+\Delta \mathrm{S}+\Delta \mathrm{PS}+\Delta \mathrm{TK}+\Delta \mathrm{A}=\mathrm{F}_{\text {in }}-\mathrm{F}_{\text {out }},
$$

with $\Delta$ in (Eq. (1)) representing daily reservoir changes; while $\mathrm{F}_{\text {in }}$ and $\mathrm{F}_{\text {out }}$ represent daily exchange in and out of the system.

Biosphere 2 water system has been designed to operate in a closed mode (no water loss to the outside; $F_{\text {in }}=F_{\text {out }}=0$ ). However, water may be imported and exported - in a controlled manner and in measurable amounts - out of the Biosphere 2 system as needed; for example, in order to reduce accumulation of salts and particles inside the primary storage tank. Poorly-measurable water losses only occur whenever Biosphere 2 is operated in 'flow through', a method recently employed to lower internal atmospheric $\mathrm{CO}_{2}$ concentrations for research purposes. Water losses occur during flow-through due to large differences in relative humidity (RH) between inside and outside air. Arizona desert air at very low RH (typically $5-10 \%$ ) enters Biosphere 2, while air leaving the system (from the Rain Forest biome) has RH values well above $90 \%$. Estimates of losses due to flow-thorough are given below.

For the period analyzed in this study, Biosphere 2 was operated in all three modes described above. Specifically, water was exported gradually between 16 
October and 1 November 1995, then quickly replaced with clean well water (Total Dissolved Solids, TDS $<50 \mathrm{ppm}$ ) between 1 and 5 November 1995 in order to reduce TDS levels (see Fig. 2). Biosphere 2 was operated in flow-through mode on average every other day, for 7-10-h intervals overnight. For the rest of the period of study the Biosphere 2 water system was operated in a closed mode.

For simplicity, while we accounted for imported and exported water amounts in our model calculations, we ignored water losses due to flow-through. To estimate the error associated with the latter approximation, we calculated that a 24-h continuous ventilation of the Biosphere 2 atmosphere, given an average flow velocity of $5000 \mathrm{cfm}$ (typical of Biosphere 2 flow-through mode) and a water vapor density of about $30 \mathrm{~g} \mathrm{~m}^{-3}$ in the Rain Forest biome (an upper limit to internal water vapor concentrations, corresponding to saturated air at $30^{\circ} \mathrm{C}$ and $88.4 \mathrm{kPa}$ pressure) would produce a water loss of roughly $5 \times 10^{3} 1 \mathrm{day}^{-1}$. This loss corresponds to about twice the mean atmospheric water content, but is still only $0.5 \%$ of total Biosphere 2 water. For the period analyzed, we calculate that this loss was at most $6 \%$ of mean daily condensate production (see section below).

We next discuss data collected in situ relative to the period of 7 October-13 December 1995 and use them to further characterize the terms in Eq. (1). Water data were collected daily using both mechanical meters and sensors. These include a set of Metron-Spectrum (Metron-Farnier, Boulder $\mathrm{CO}$ ) and Signet (GeorgeFisher, Logan UT) electronic flow meters of various sizes, with a typical reading in the range of $1-4501 \mathrm{~min}^{-1}$ and an accuracy of $0.01 \%$.

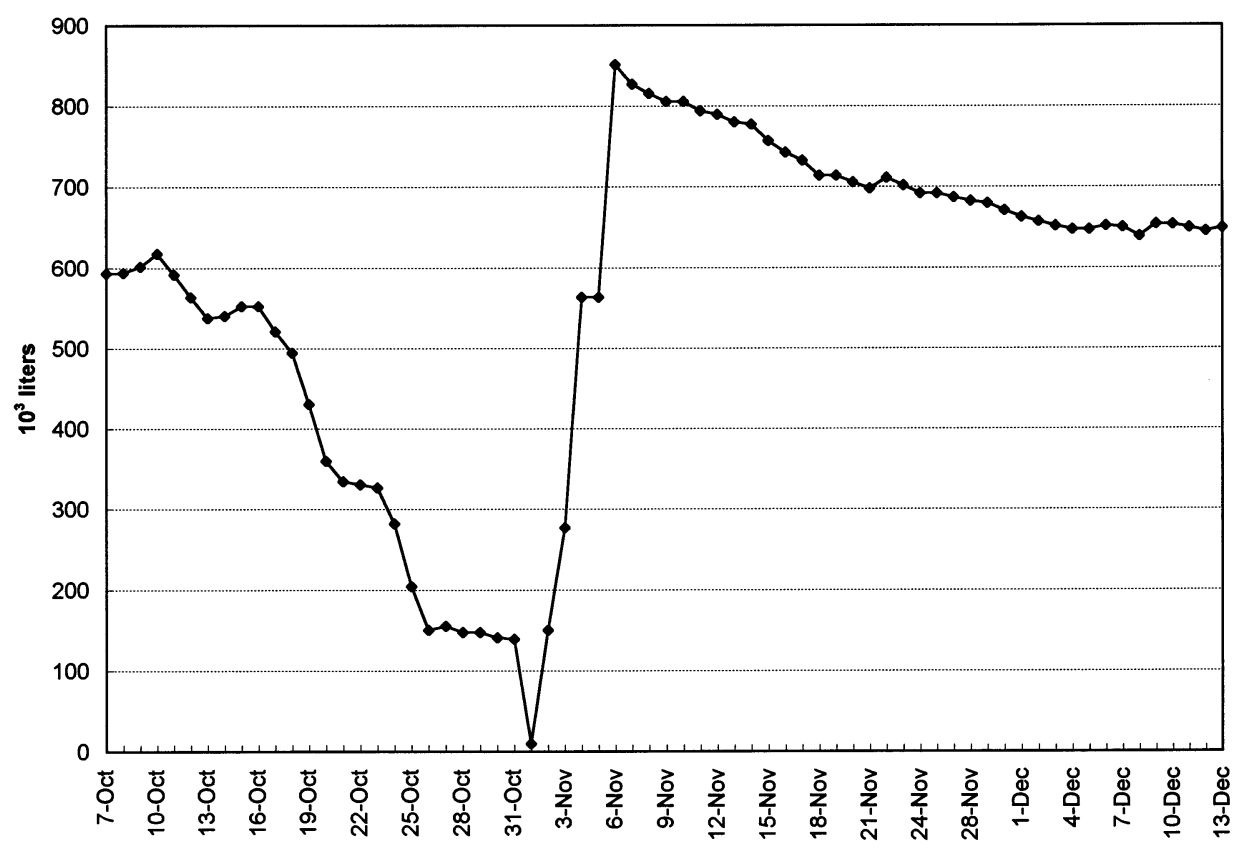

Fig. 2. Water levels in the primary storage for the period analyzed in 1995. 


\section{Data analysis: mean daily flows between reservoirs}

\subsection{Primary storage}

Water level data in the primary storage were monitored continuously with an Lamdahl DST ultrasonic sensor (Lamdahl Instruments, Logan, UT), reporting to a central database every $15 \mathrm{~min}$. The instrument specified precision is $0.02 \%$ of daily mean readings, corresponding to an error of about 1001 per day. Fig. 2 shows the water level in the PS tank as a function of time. During the period analyzed water was exported to the outside until the tank was almost empty and external well water was used to quickly replenish the tank to capacity.

The primary storage tank is central to the closure of Biosphere 2 water cycle. Water enters the primary storage as subsoil drainage, collected from the bottom of each biome's soil profile; and includes all water leakages in the system, which flow back to primary storage along the Biosphere 2 basement floor. Daily total subsoil drainage is usually in the order of $10^{3} 1$ (drainage from the rain forest soils, a major source of the Biosphere 2 total, can be as high as $3 \times 10^{3} 1 \mathrm{day}^{-1}$ ). Water returns to the rest of the Biosphere 2 system via reverse osmosis (RO) production, used to reduce the concentration of total dissolved solids in primary storage water. An RO machine (Watertech, Tucson AZ) with a $41 \mathrm{~min}^{-1}$ flow production capacity is currently used for the purpose. RO water is delivered to a system of collection tanks in both the agricultural and wilderness biome areas. Mean daily RO production was about $4 \times 10^{3} 1$ day $^{-1}$ for the period analyzed. Given the large capacity of the primary storage reservoir, the residence time of the water stored there is thus of the order of months.

\subsection{Condensate tanks}

Six tanks in the wilderness area basement and eight tanks in the intensive agricultural biome (IAB) basement collect water available for irrigation and rain applications. Three additional tanks in the IAB, originally intended for storage of soil drainage water, may be made available as needed for condensate storage. Each tank has a $9.5 \times 10^{3} 1$ volume, for a total Biosphere 2 condensate water capacity of $1.6 \times 10^{5} 1$. For the purpose of a global water budget all these tanks were represented by one ideal 'condensate tank' (TK) in Eq. (1).

The condensate tank reservoir represents the dynamical pump of the water system. Condensate water moves quickly in and out of this reservoir: it is used on a daily basis for both rain and irrigation of all biomes and to supply the ocean reservoir with water lost through evaporation. It is produced by reverse osmosis, as discussed; as well as by an extensive network of air handlers units (AHU), which constitute Biosphere 2 climate control process (Zabel et al., 1999). Fig. 3 shows condensate tank levels for the period analyzed. Fig. 4 illustrates daily changes in tank level $(\Delta \mathrm{TK})$ and water fluxes in and out of this reservoir. Rain water and condensate production fluxes were in the order of $10^{4} 1$ per day. Water supplied to the ocean reservoir was comparable to that produced by $\mathrm{RO}$ and both were about 


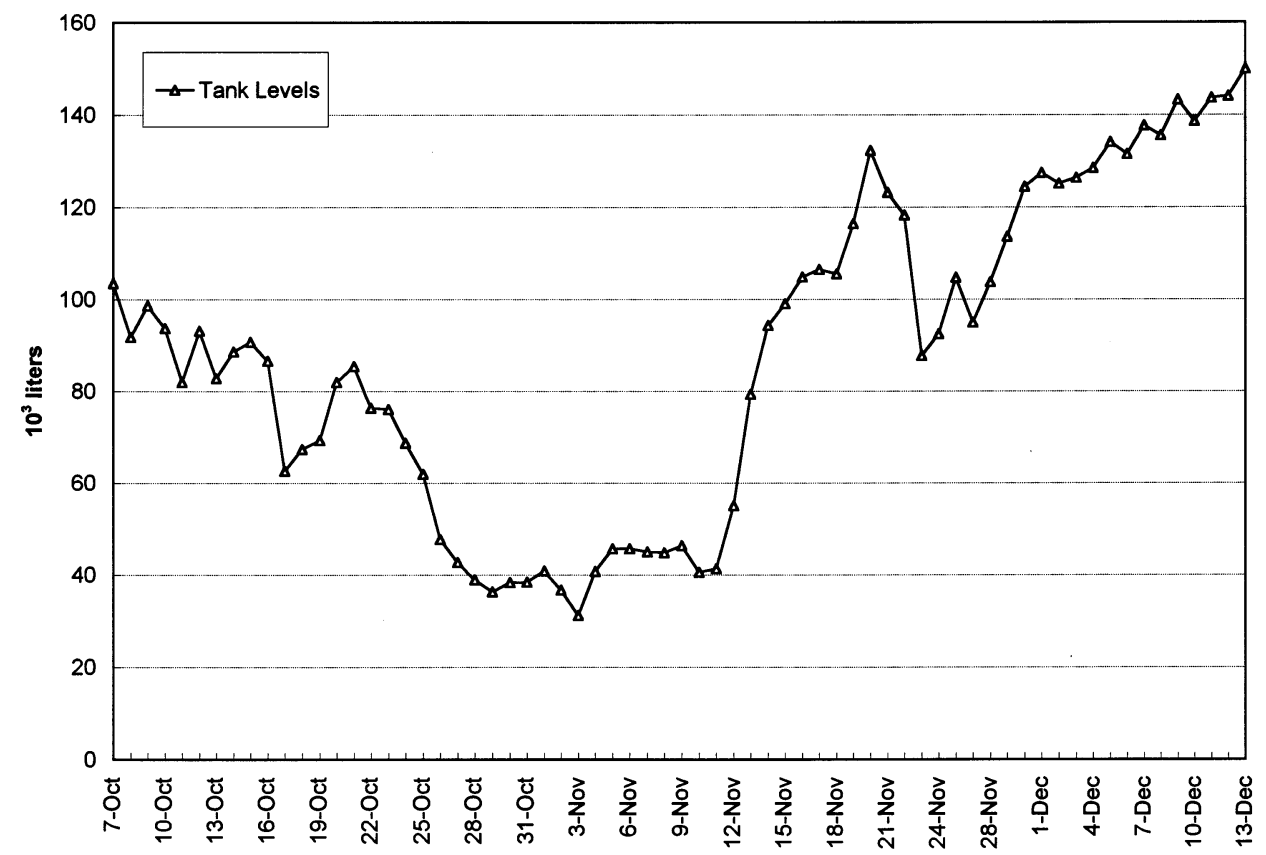

Fig. 3. Water levels in the condensate tanks for the period analyzed.

one-half of AHU production. Daily oscillations in the condensate reservoir were about $5 \times 10^{3} 1$. They were well correlated with rain water fluxes (Fig. 4b), which had the largest day-to-day variation among the water fluxes analyzed.

\subsection{Biosphere 2 ocean-marsh and atmospheric reservoirs}

The ocean-marsh and atmospheric reservoirs were extremely simplified in our model. Mean daily fluxes of condensate water to the ocean-marsh reservoir were $3.3 \times 10^{3} 1$ for the period analyzed. We assumed in our calculations that this supply equaled evaporative losses from the ocean plus evapotranspiration losses from the marsh area, or $\Delta \mathrm{O} \sim 0$. Although this assumption is not true on a daily basis, it roughly applies to monthly mean calculations: the Biosphere 2 marsh-ocean water level is in fact stable over the long term (Atkinson et al., 1999), with monthly fluctuations typically no larger than $9 \times 10^{3} 1$. Errors in daily mean evaporative fluxes under our simplification are then of roughly 3001 per day, or less than $10 \%$.

The atmospheric water reservoir is not only small compared to the others analyzed here, as previously discussed, but its daily mean fluctuations are also small compared to other fluxes. Table 2 shows estimates of mean water content in the atmosphere for each biome, as calculated from temperature and relative humidity sensors of Biosphere 2. Fig. 5 shows, as an example, typical fluctuations of atmospheric water vapor in the desert biome. 

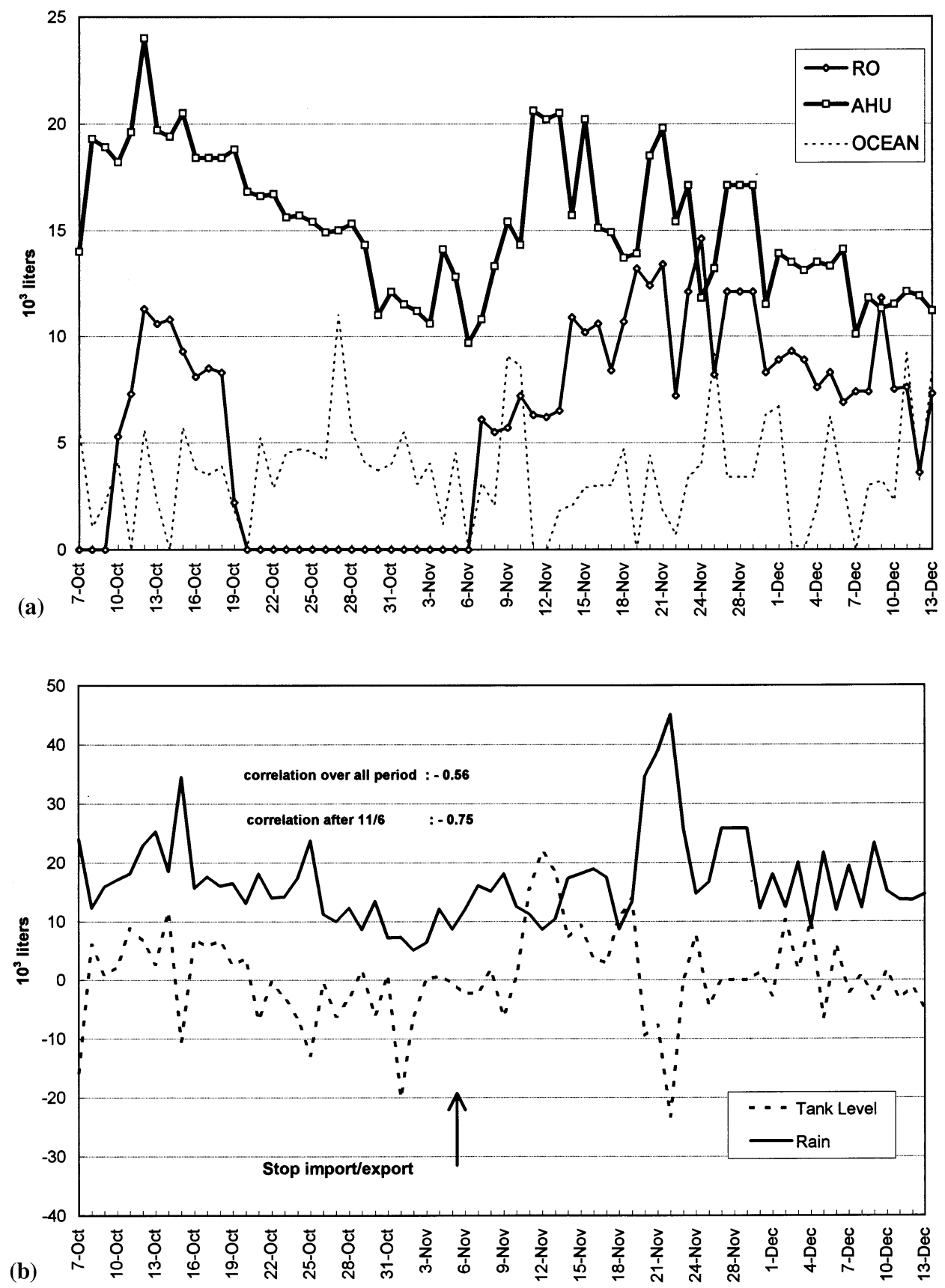

Fig. 4. (a) Usage of condensate water for the period analyzed; (b) Correlation of daily fluctuations in condensate water levels and total rain applied. 
Table 2

Estimate of Biosphere 2 water vapor reservoirs, in liters

\begin{tabular}{lc}
\hline Reservoir & Size \\
\hline Rain Forest & $800 \pm 80$ \\
Savannah $^{\mathrm{a}}$ & $600 \pm 100$ \\
Desert & $200 \pm 20$ \\
IAB & $400 \pm 40$ \\
Total & $2000 \pm 200$ \\
\hline
\end{tabular}

Fluctuations of $10 \%$ are indicated. Calculations are based on temperature and relative humidity data measured during the period of study.

a Includes atmosphere over the marsh-ocean system.

\section{Water movement}

We next present results from our model calculations. In order to develop a rough assessment of soil and plant evapotranspiration using the global water budget data, we added the following relations (with reference to Fig. 1) to Eq. (1):

$$
\begin{aligned}
& \Delta \mathrm{O} \sim 0 ; \quad \Delta \mathrm{S}^{\prime}=\Delta \mathrm{S}+\Delta \mathrm{A} ; \quad \mathrm{ET}^{\prime}=\mathrm{ET}+\Delta \mathrm{A} ; \\
& \Delta \mathrm{S}^{\prime}=\mathrm{RAIN}+\mathrm{O}_{\mathrm{EV}}-\mathrm{DRNG}-\mathrm{AHU} ;
\end{aligned}
$$

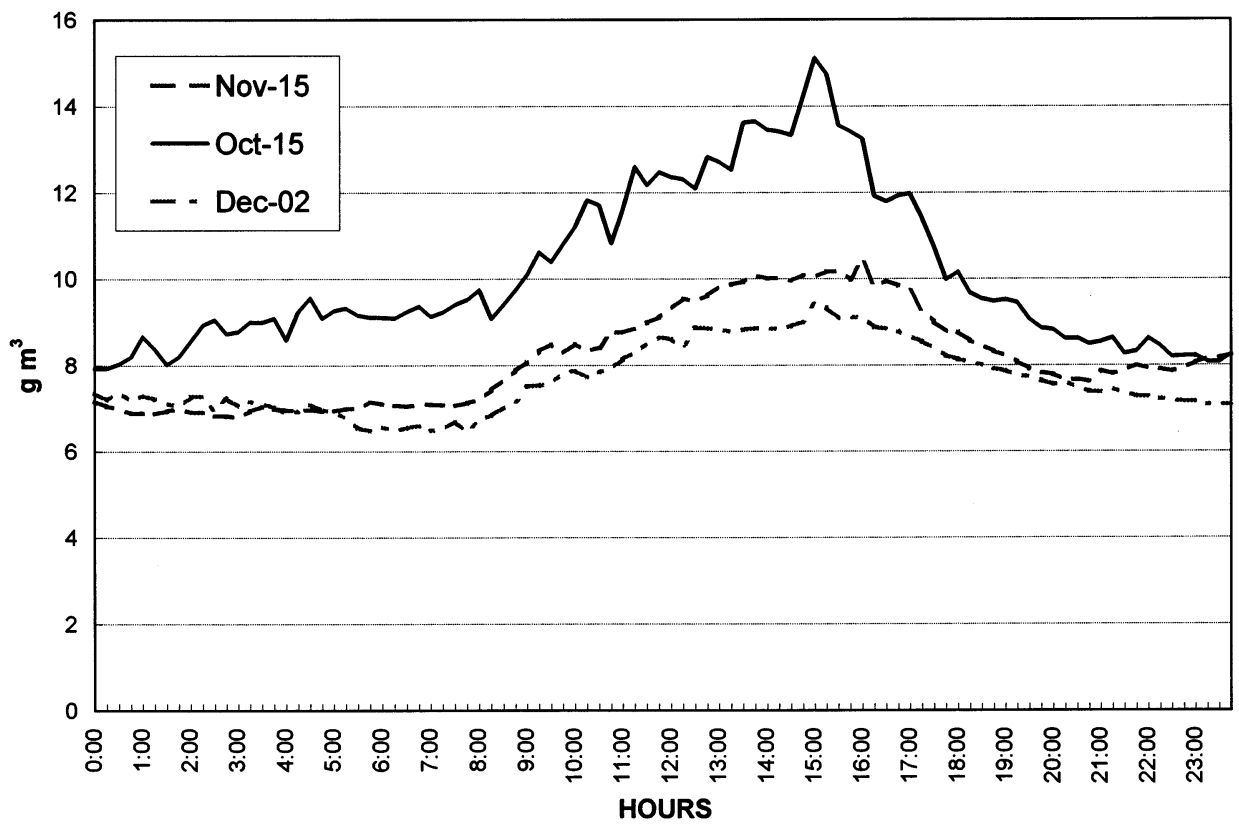

Fig. 5. Water vapor concentration in the desert biome, for typical days of the period analyzed. 


$$
\begin{aligned}
& \mathrm{ET}^{\prime}=\mathrm{AHU}-\mathrm{O}_{\mathrm{EV}} ; \\
& \Delta \mathrm{TK}=\mathrm{RO}+\mathrm{AHU}-\mathrm{RAIN}-\mathrm{O}_{\mathrm{ET}} ;
\end{aligned}
$$

where ET is whole system evapotranspiration; RAIN is total rain and irrigation applied; $\mathrm{O}_{\mathrm{ET}}$ is ocean plus marsh evapotranspiration; DRNG represents overall soil drainage; and AHU is total air handlers production. The variables $\mathrm{S}^{\prime}$ and $\mathrm{ET}^{\prime}$ have been introduced to simplify soil balance and evapotranspiration calculations, being needed for lack of knowledge, a priori, of the relative magnitude of these latter two terms and the daily atmospheric balance, $\Delta \mathrm{A}$. Using the daily data discussed above, i.e. by assessing the terms $\Delta \mathrm{P} ; \Delta \mathrm{TK} ; \Sigma \mathrm{W}$; the unknown $\Delta \mathrm{S}^{\prime}$ can be easily obtained from (Eq. (1)); soil drainage, DRNG, can then be derived from (Eq. (2b)) by knowing the terms RAIN, $\mathrm{O}_{\mathrm{EV}}$ and AHU. The sum of whole-system evapotranspiration and atmospheric changes can be calculated from AHU and ocean evaporation independently, using (Eq. (2c)). We found that over the period analyzed $\Delta \mathrm{A} \ll \Delta \mathrm{S}^{\prime} \ll \mathrm{ET}^{\prime}$. This implies in particular that whole-system evapotranspiration, ET, can be calculated, as a first approximation, from air handlers production and ocean evaporation data alone. By assuming $\Delta \mathrm{A} \sim 0$, relative errors in the estimated ET no greater than a few percentage points are generated. Table 3 lists our results relative to the 8 weeks analyzed, providing 2 monthly totals and an overall average. The grand mean average indicated in this table should be interpreted with caution, as it covers two different Biosphere 2 operation modes: water flow through; and mass-closure. It is nonetheless remarkable that important features in the calculated data exhibit a rather constant behavior. For example, with reference to rain water applied, mean air handlers production was $80-90 \%$ of the daily amount, while whole-system ET was consistently about two-thirds of it. While the mechanical components of the system exhibited wide fluctuations around their mean values, as a consequence of changes in the day-to-day operation of Biosphere 2 as a mechanical system, those fluxes controlled by natural components were more stable around the calculated means.

From Tables 1 and 2 it is possible to calculate turnover times for each of the five major reservoirs: PS, TK, O, A and S. These estimates, obtained by dividing mean reservoir size by mean flow out, are only meant to offer a first numerical assessment of the timescales involved with water movement inside Biosphere 2. Steady-state conditions are usually required in order to correctly interpret these results (Tubiello and Oppenheimer, 1995). In fact, it is reasonable to assume no change in reservoir size only in the mean sense (see Table 3). Although the two periods analyzed were very different in terms of water management, the values of the timescales were found to be rather stable. As shown in Table 4, they ranged from a period of a few hours (atmosphere), to days (condensate water tanks), to months (primary storage and soil), to several years (ocean).

\subsection{Three water cycles in Biosphere 2}

Water in the condensate tank reservoir is readily used for rain and irrigation, as discussed, being constantly replaced by air handler's production. Its fast turnover 
Table 3

Mean \pm SD of the main water fluxes (units: $10^{3}$ liters) calculated for Biosphere 2

\begin{tabular}{|c|c|c|c|c|c|c|c|c|}
\hline Period & RO & $\Delta \mathrm{PS}$ & $\Delta \mathrm{TK}$ & RAIN & AHU & OCEAN & ET & DRNG \\
\hline October & $4.1 \pm 4.6$ & $4.6 \pm 4.1$ & $-3.0 \pm 9.3$ & $18.3 \pm 5.5$ & $18.0 \pm 2.3$ & $3.3 \pm 0.3^{\mathrm{a}}$ & $14.7 \pm 3.3$ & $5.2 \pm 12.8$ \\
\hline November & $9.0 \pm 2.7$ & $-4.8 \pm 4.3$ & $2.8 \pm 9.4$ & $18.2 \pm 8.2$ & $14.7 \pm 3.0$ & $3.5 \pm 0.4$ & $11.1 \pm 3.6$ & $4.2 \pm 9.4$ \\
\hline Grand mean & $7.2 \pm 4.2$ & $-2.0 \pm 3.1$ & $-0.7 \pm 9.7$ & $18.3 \pm 7.3$ & $15.9 \pm 3.2$ & $3.4 \pm 0.4$ & $12.4 \pm 4.5$ & $4.6 \pm 10.6$ \\
\hline
\end{tabular}

${ }^{a}$ Assuming 10\% error in daily mean quantities. 
Table 4

Reservoir turnover times inside Biosphere 2

\begin{tabular}{llllll}
\hline Period & ATMO $^{\mathrm{a}}$ & TK $^{\mathrm{a}}$ & PS $^{\mathrm{a}}$ & SOIL & OCEAN \\
\hline October & $2.7 \mathrm{~h}$ & 4.7 days & 76.0 days & 50.3 days & 1212 days \\
November & $3.3 \mathrm{~h}$ & 4.6 days & 77.9 days & 65.4 days & 1111 days \\
Mean & $3.0 \mathrm{~h}$ & 4.7 days & 77.0 days & 57.8 days & 1162 days \\
\hline
\end{tabular}

${ }^{\text {a }} \mathrm{ATMO}=$ atmosphere; $\mathrm{TK}=$ condensate tanks; $\mathrm{PS}=$ primary storage.

time, about 5 days and large size make it the heart of Biosphere 2 global water cycle. What happens to this water as it moves around the system? Fig. 6 shows the three main cycles that best describe such movement. Our calculations suggest the following. Of the water used on a daily basis, or roughly $22 \times 10^{3} 1$, comprising rain $\left(18.5 \times 10^{3}\right)$ and ocean supply $\left(3.5 \times 10^{3}\right)$, about $60 \%$ is returned through the

\section{BIOSPHERE 2 WATER CYCLES}

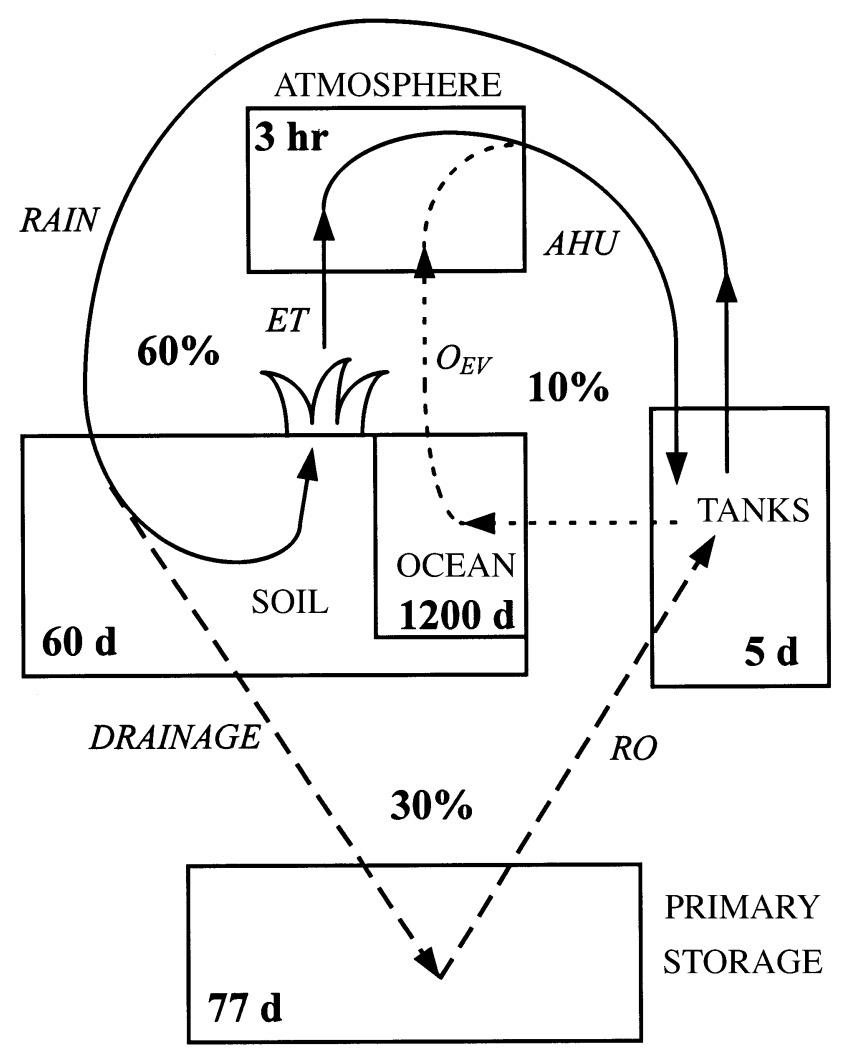

Fig. 6. Three major water cycles as suggested by the data analyzed. Indicated are turnover times in each reservoir. 
atmosphere over a period of roughly a month, via soil and plant evapotranspiration. This estimate assumes that the water transpired resided mainly in Biosphere 2 loamy topsoil. This layer is on average $1 \mathrm{~m}$ deep throughout the biomes, corresponding to a volume of about $7 \times 10^{3} \mathrm{~m}^{3}$ (Scalborough, 1994). At an average $10 \%$ volumetric water content (accounting for rain forest and savannah soils at field capacity and for dry Desert and IAB soils - this area was not intensively irrigated during the period analyzed) this corresponds to a $5 \times 10^{5} 1$ water reservoir. Dividing this amount by the mean ET flux yields a turnover time of 33 days. Once this water is in the atmosphere, it is immediately (few hours turnover time) returned to the condensate tanks via air handler production.

Rain water applied that is not evaporated, or about $30 \%$ of the water used daily, drains through the soil profile to primary storage. We estimate that it may take 6 months to a year (at most 300 days for overall drainage and 70 days to leave the primary storage via RO production) to return to the condensate tanks. Finally, the remaining $10 \%$ of the water used daily is supplied to the ocean-marsh. It takes about 3 years before it is returned to the condensate water reservoir.

Finally, it should be noticed that the data used for this study were collected in the fall-winter season. Increases in evapotranspiration rates, possibly leading to faster turnover times then calculated in this work, should be expected in the summer periods due to higher solar loading.

\subsection{Model applications: response to a pollutant infiltration}

The simple model we have developed is not only useful to assess mean daily amounts of water moving inside Biosphere 2, but can be easily applied to calculating perturbation response times in all of the five major reservoirs analyzed. Specifically, if a given tracer (a pollutant, for example) were to invade one of the reservoirs, what dynamics would characterize its time dependent concentration? How would it spread around the Biosphere 2 water system? We considered the case of a chemically stable tracer added to the condensate tank reservoir. We assumed that the pollutant could not leave the soil or ocean reservoirs by evaporation and that return $\mathrm{RO}$ water from primary storage into the condensate tanks was free of the pollutant. Physical-chemical interactions between the pollutant and soil particles were not modeled. Assuming steady-state water fluxes among reservoirs, Eqs. (2a), (2b), (2c) and (2d) can be formalized into a system of linear differential equations and solved for the tracer concentration. We only present here the results of our calculations without discussing the mathematical details. The interested reader is referred elsewhere for a discussion of perturbation response times and their associated dynamics (e.g. Lasaga, 1980). Fig. 7 shows the time-dependent tracer concentration in the condensate tanks and its redistribution among the other reservoirs in the system. Results show that under water flow conditions similar to those found in this study, more than $90 \%$ of the pollutant would be washed out of the condensate tanks in a few days, accumulating at first in the soil and ocean systems. In about a months time, the pollutant would disappear completely from the condensate tanks, reaching a stable concentration $(14 \%)$ in the ocean. Finally at steady-state, $85 \%$ of the pollutant would be found trapped in the primary storage tank and only about $1 \%$ would remain in the soils. 


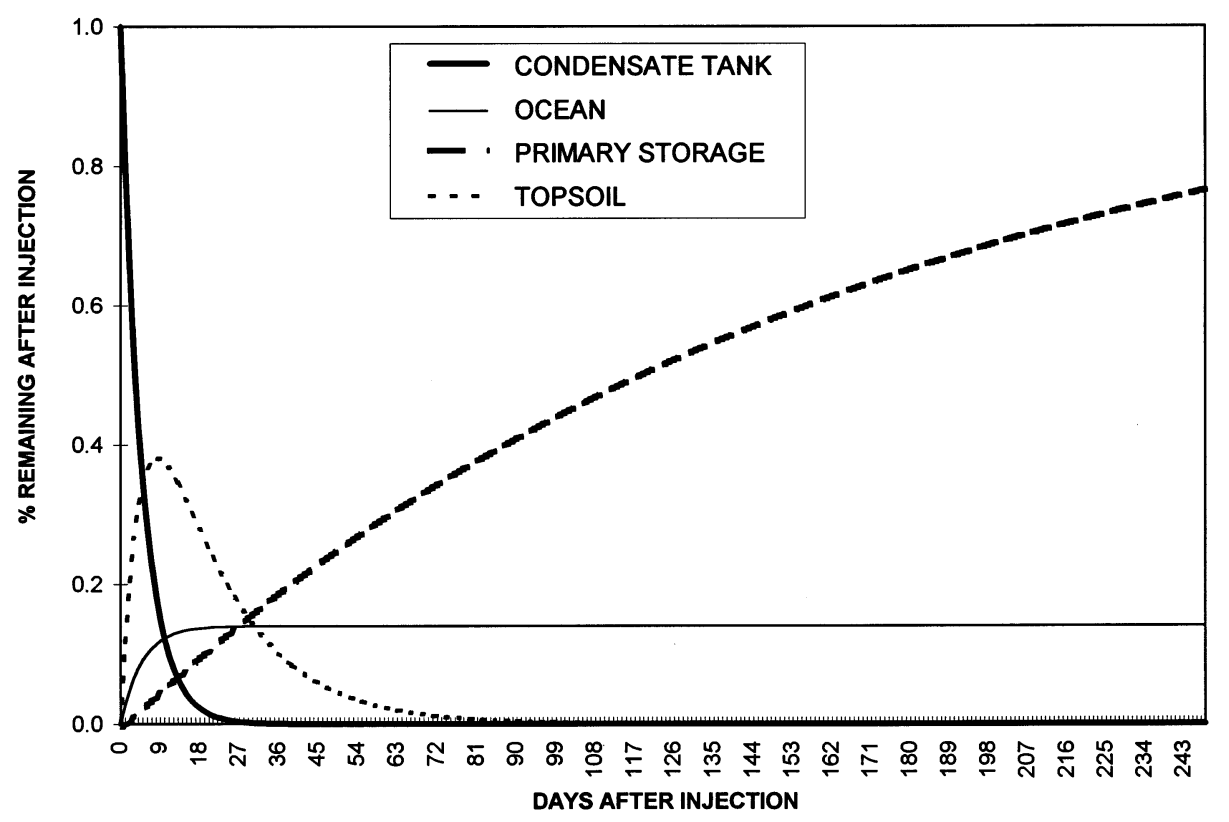

Fig. 7. Response to a pollutant added to the condensate water tanks. As the tracer leaves the reservoir mainly as rain, it initially accumulates in the soil profile and it is later redistributed to the ocean via supply of condensate tank water and to the primary storage through soil drainage.

\section{Conclusions}

We have shown that it is possible to characterize the global water cycle of Biosphere 2 with the aid of a simple model, assuming that only five reservoirs significantly contribute to the daily water budget. On average, about $20 \times 10^{3} 1$ of water circulate in Biosphere 2 on a daily basis. This water follows three virtually separate cycles inside the system, with timescales that range from hours to several years and which are associated with both natural - soil and plant evapotranspiration, ocean evaporation, soil drainage - and mechanical processes inside Biosphere 2 (condensate production, reverse osmosis, rain and irrigation). In particular, the coupling of whole-system evapotranspiration to air handlers production and rain applications provides for a vigorous turnover of water in the condensate tanks, as shown by both data and model calculations.

Characterizing water movements inside Biosphere 2 is of great importance for the future maintenance of this facility and planning of its research activities. Understanding the dynamics of its water cycle will help us optimize water production and use. In addition, first-order estimates of reservoir turnover times like the ones briefly described in this work will help trace chemical species (both pollutants and tracers of importance to maintenance and research teams) as they move inside Biosphere 2. Finally, our current efforts indicate that the mass-conservation equations developed globally herein can be applied at a smaller scale, to calculate water budgets for the single biomes of Biosphere 2 . 


\section{Acknowledgements}

We wish to thank the Biosphere 2 research and maintenance staff for support. Comments from two anonymous reviewers contributed to improve the quality of this work.

\section{References}

Atkinson, M., Barnett, H., Anderson, H., Langdon, C., Carpenter, S.J., McConnaughey, T., Hochberg, E., Smith, M., Marino, B.D.V., 1999. The Biosphere 2 coral reef biome. Ecol. Eng. 13, 147-172.

Dempster, W.F., 1992. Water systems of Biosphere 2. Paper presented to the International Conference on Life Support and Biospherics, University of Alabama, Huntsville, AL, 20 February, 1992. Space Biosphere Ventures, Oracle, AZ.

Lasaga, A.C., 1980. The kinetic treatment of geochemical cycles. Geoch. Cosmochim. Acta 44, 815-828.

Leigh, L., Burgess, A, Marino, B.D.V., Wei, Y.D., 1999. Tropical rainforest biome of Biosphere 2: Structure, composition and results of the first 2 years of operation. Ecol. Eng. 13, 65-93.

Marino, B.D.V., Mahato, T., Druit, J.W., Leigh, L., Lin, G., Russell, R.M., Tubiello, F.N., 1999. The agricultural biome of Biosphere 2: Structure composition and function. Ecol. Eng. 13, 199-234.

Petersen, J., Haberstock, A.E., Siccama, T.G., Vogt, C.A., Vogt, D.J., Tusting, B.L., 1992. The making of Biosphere 2. Rest. Manage. Notes 10 (2), 158-168.

Scalborough, R., 1994. Soils Final Report, Biosphere 2. Internal Paper, Space Biosphere Ventures, Oracle, AZ.

Tubiello, F.N., Oppenheimer, M., 1995. Anthropogenic $\mathrm{CO}_{2}$ and impulse-response functions. Geophys. Res. Let. 22 (4), 413-416.

Zabel, B., Hawes, P., Stuart, H., Marino, B.D.V., 1999. Construction and engineering of a created environment: Overview of the Biosphere 2 closed system. Ecol. Eng. 13, 43-63. 